\title{
Early detection and efficient therapy of cardiac angiosarcoma due to routine transesophageal echocardiography after cerebrovascular stroke
}

\author{
Dirk Vogelgesang' \\ Johannes B Dahm² \\ Holm Großmann ${ }^{3}$ \\ Andre Hippe ${ }^{4}$ \\ Astrid Hummel ${ }^{5}$ \\ Christian Lotze ${ }^{6}$ \\ Silke Vogelgesang ${ }^{7}$

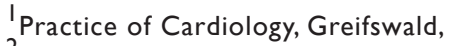 \\ ${ }^{2}$ Practice of Cardiology, Goettingen, \\ ${ }^{3}$ Department of Cardiovascular \\ Surgery, Herzzentrum Karlsburg, \\ ${ }^{4}$ Department of Neurology, \\ ${ }^{5}$ Department of Cardiology, \\ ${ }^{6}$ Department of Haematology and \\ Oncology, ${ }^{7}$ Department of Pathology, \\ University of Greifswald, Greifswald, \\ Germany
}

\begin{abstract}
Primary malignant cardiac tumors (cardiac angiosarcomas) are exceedingly rare. Since there are initially nonspecific or missing symptoms, these tumors are usually diagnosed only in an advanced, often incurable stage, after the large tumor mass elicits hemodynamic obstructive symptoms. A 59-year-old female presented with symptoms of cerebral ischemia. A computed tomography (CT) scan showed changes suggestive of stroke. Transesophageal echocardiography revealed an inhomogeneous, medium-echogenic, floating mass at the roof of the left atrium near the mouth of the right upper pulmonary vein, indicative of a thrombus. At surgery, a solitary tumor was completely enucleated. Histologically, cardiac angiosarcoma was diagnosed. The patient received adjuvant chemotherapy and was free of symptoms and recurrence of disease at 14 months follow-up. Due to the fortuitous appearance of clinical signs indicative of stroke, cardiac angiosarcoma was diagnosed and effectively treated at an early, nonmetastatic, and therefore potentially curable stage. Although cardiac angiosarcoma is a rare disease, it should be taken into consideration as a potential cause of cerebral embolic disease.
\end{abstract}

Keywords: cardiac angiosarcoma, stroke, embolism

\section{Introduction}

Cardioembolic stroke is a major and frequent complication of various cardiac diseases. By far the most common cardiac sources of embolic stroke include atrial fibrillation, ischemic heart disease, mechanical prosthetic valves, and rheumatic heart disease. Nonischemic dilated cardiomyopathy, infective endocarditis, thrombotic vegetations, myxomatous mitral valve, paradoxical embolism, atrial septal aneurism, mitral anular calcification, calcific aortic stenosis, and left atrial cardiac tumors (ie, myxoma) are further causes of cardioembolic disease (Hart 1992). In addition, malignant primary cardiac tumors are an exceedingly rare cause of cardioembolic stroke. Primary cardiac tumors themselves are generally infrequent. Among these tumors, benign lesions (ie, myxomas or rhabdomyomas) predominate. Within the malignancies, sarcomas and especially angiosarcomas represent the most common entities (Mayer et al 2007; Yu et al 2007). Here we present a case of a cardiac angiosarcoma that was diagnosed and effectively treated at an early stage due to clinical signs of stroke.

\section{Case report}

A 59-year-old woman was admitted to the hospital due to a sudden onset of occipital headache, nausea and dizziness. A cerebral computed tomography (CT) scan confirmed a cerebellar (embolic) ischemic stroke. The patient was transmitted to the stroke unit, and a routine diagnostic procedure to evaluate the origin of the embolism was carried out. A duplex-sonographic scan of the brain-supplying arteries revealed 
no abnormalities. Atrial fibrillation as a potential source of cardiac emboli could not be demonstrated during 24-hour Holter monitoring. Transesophageal echocardiography (TEE) revealed a $1.5 \mathrm{~cm}$ floating mass that originated from the roof of the left atrium, near the mouth of right upper pulmonary vein (Figure 1), strongly suggestive of a floating thrombus. The remaining cardiac structures were free of abnormalities. The patient was immediately sent to cardiac surgery, where the operation was performed the same day. A polycystic villous tumor with a smooth, fatty consistency around a hard core was enucleated and removed in toto.

Microscopically, a tumor with broad necrosis lined by a thin rim of densely packed tumor cells was diagnosed. These tumor cells were highly polymorphic and showed rounded or spindle-shaped nuclei with prominent nucleoli. Single multinucleated giant cells also could be observed. The mitotic rate was as high as 5 mitoses per high-power field, including atypical mitotic figures. Focally, the tumor showed little vascular channels with some erythrocytes within the lumina (Figure 2a).

Immunohistochemically, tumor cells strongly expressed endothelial markers such as factor XIII, CD31, CD34, as well as vimentin (Figure 2b). Cytokeratins, desmin and smooth muscle actin were negative. Reaction with MIB-1 revealed a high proliferation rate, with up to $30 \%$ of tumor cells positive for MIB-1 (Figure 2c). The tumor was diagnosed as a poorly differentiated angiosarcoma of the left atrium.

Following surgical removal of the tumor, the patient was moved to the Department of Oncology. With additional diagnostic procedures including $\mathrm{CT}$ and magnetic resonance imaging (MRI), a systemic or metastatic malignancy was ruled out. The patient was given an adjuvant anthracyclinecontaining chemotherapy and discharged on day 23 after surgery.

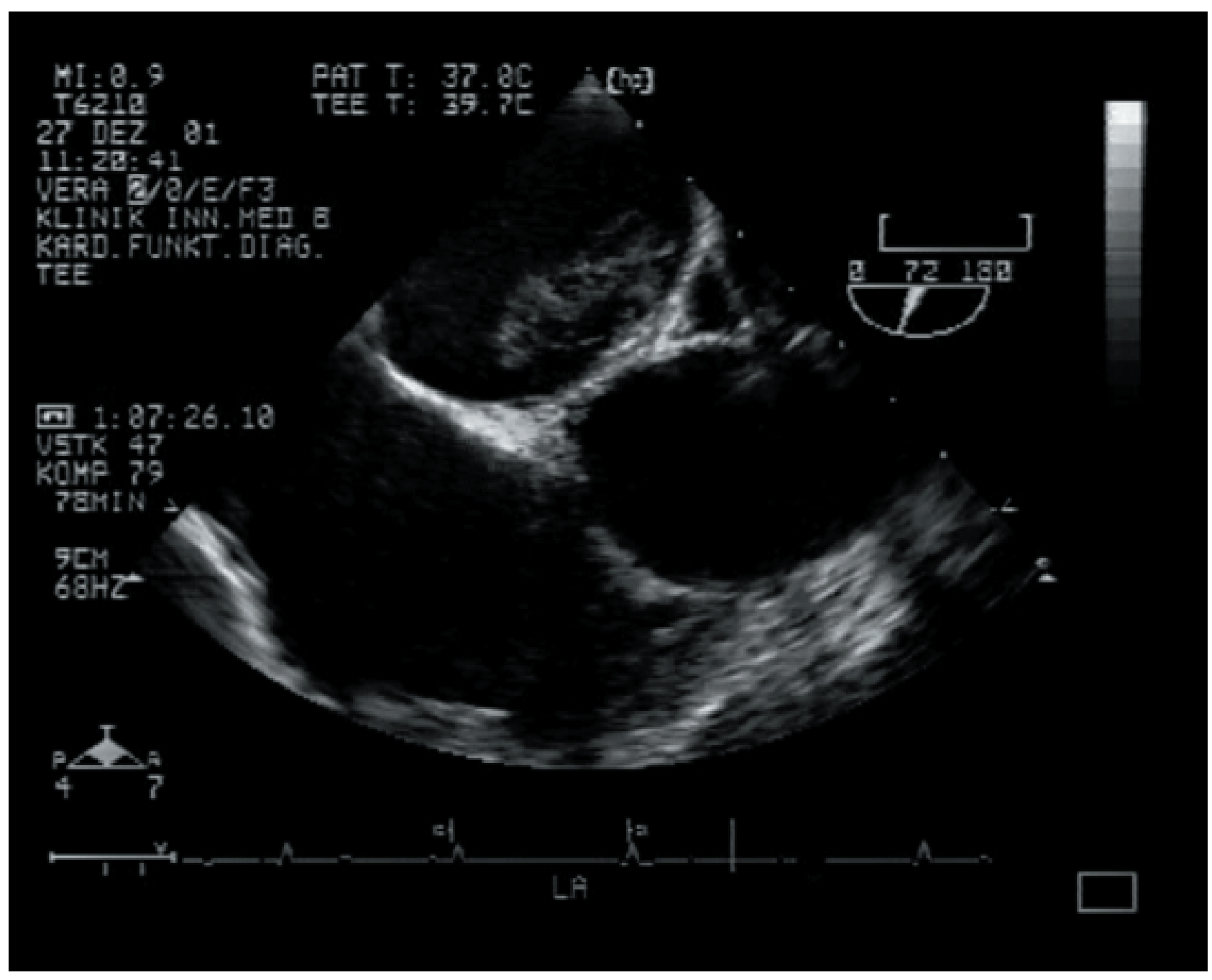

Figure I Transesophageal echocardiography showing a floating mass in the left atrium. 


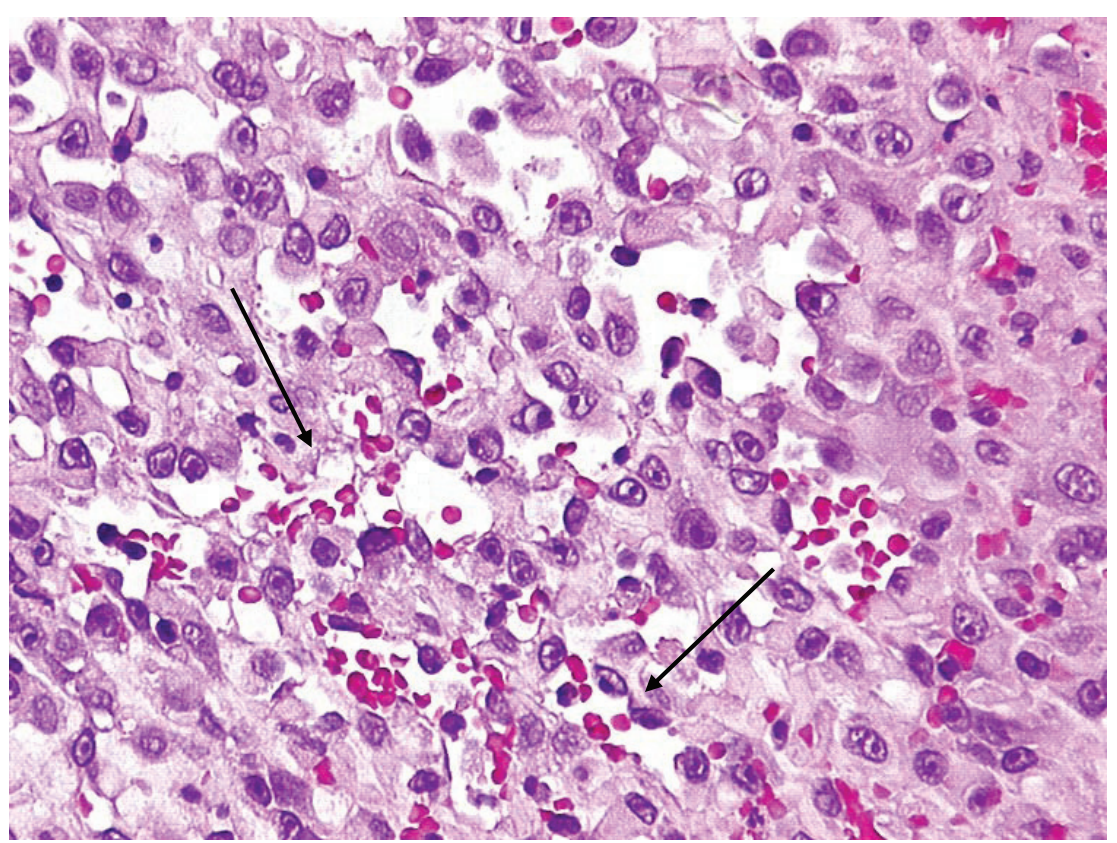

Figure 2a Microscopic analysis of a specimen from the atrial mass disclosed highly polymorphic tumor cells and vascular channels (arrow) with erythrocytes (H\&E, Original magnification $\times 200$ ).

Routine follow-up including CT, MRI, and TEE at 6 and 12 months revealed no evidence of metastastic or recurrent disease, and the patient was free of symptoms at 14 months post-surgery.

\section{Discussion}

Primary tumors of the heart are rare, being found in less than $0.3 \%$ of autopsies. The incidence of these tumors has been reported to be $0.001 \%$ to $0.03 \%$ (Glancy et al 1968). Cardiac

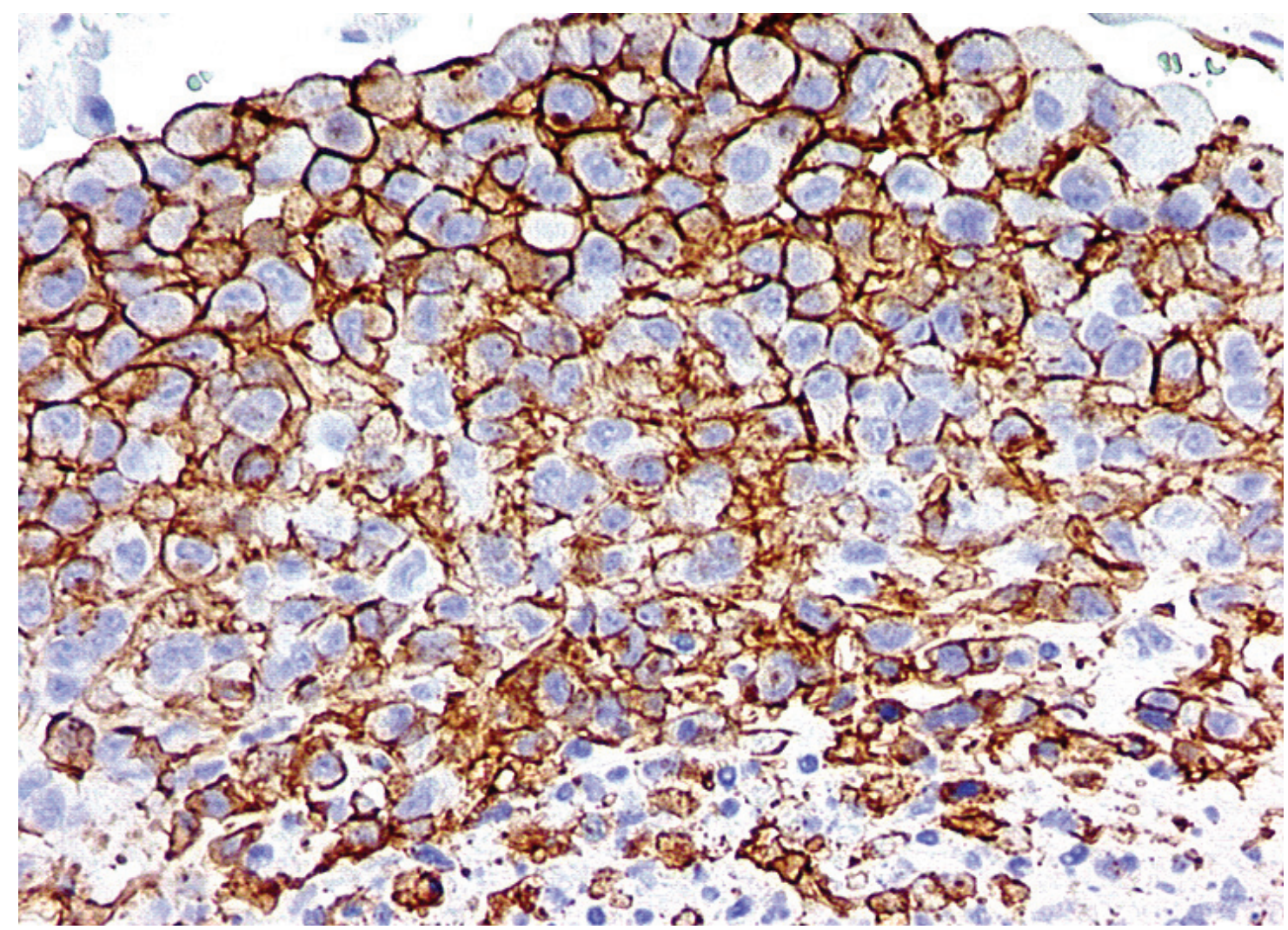

Figure 2b Immunohistochemical expression of CD3I (Brown colour, original magnification $\times 200$ ). 


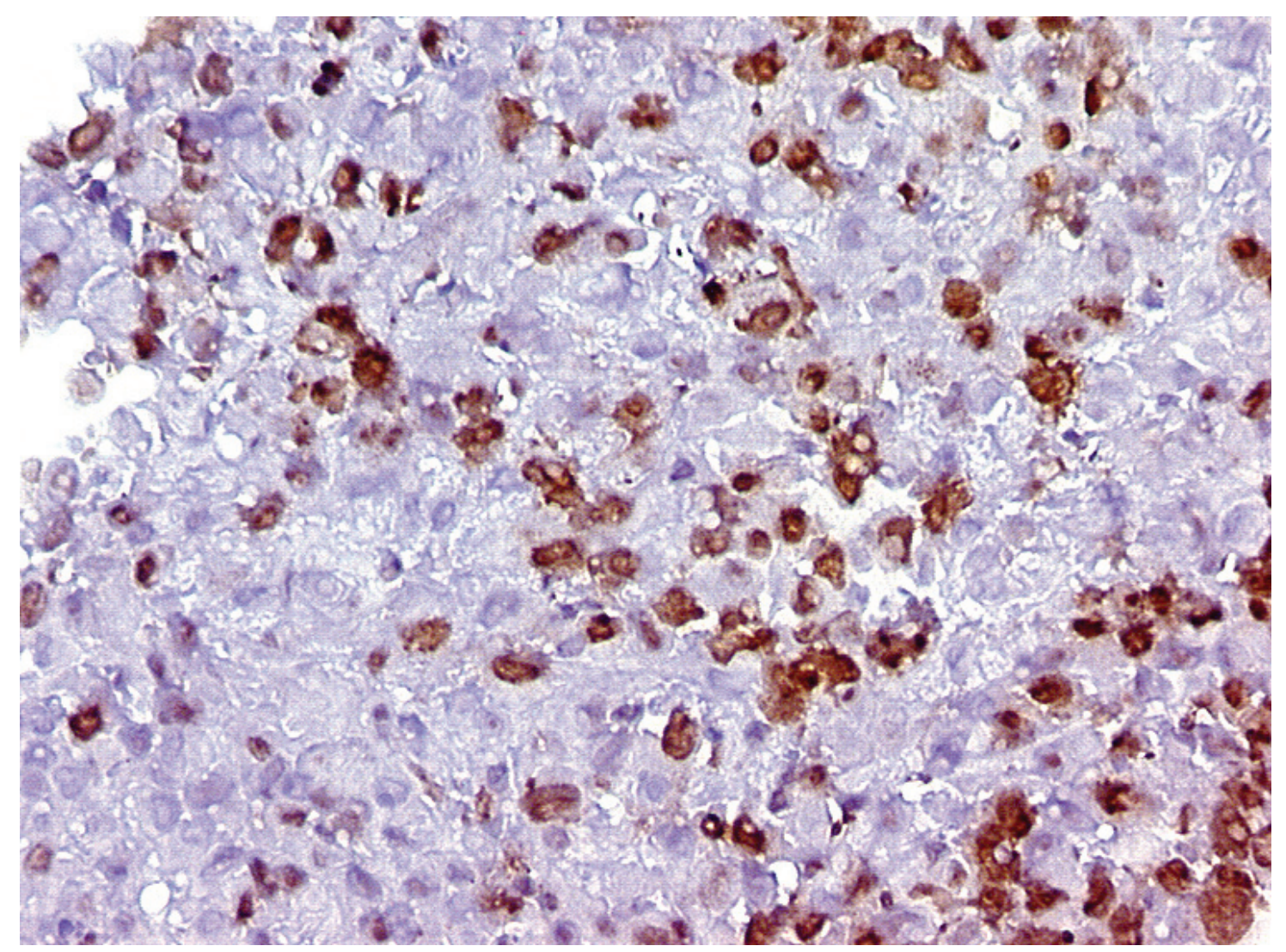

Figure 2c High proliferation index with MIB-I (Brown coloured nuclei, original magnification $\times 200$ ).

angiosarcoma accounts for less than $10 \%$ of all primary tumors of the heart (McAllister 1979; Putnam et al 1991; Burke and Virmani 1996; Yu et al 2007), but it is the most frequent malignant primary tumor of the heart with a relative incidence of about 33\% in adults (Allard et al 1995; NeragiMiandoab et al 2007). This tumor usually originates in the right atrium. The majority of patients are middle-aged, with men being at higher risk than women (Janigan et al 1986; Hart 1992; Neragi-Miandoab et al 2007). Because of the presence of multiple metastases at the time of diagnosis, the tumor is associated with a very poor prognosis (Janigan et al 1986; Putnam et al 1991). Because of its rarity, no generally applicable guidelines for treatment have been established. Surgery, adjuvant chemotherapy and radiation have been used, but despite these aggressive therapeutic strategies, most patients die within one year after diagnosis (Mayer et al 2007). Although the usual cause of death is congestive heart failure, early cardiac transplantation is not recommended because of the frequent presence of metastases at time of diagnosis (Burke et al 1992; Reardon et al 2006).

Tumors of the heart result in a wide range of clinical signs and symptoms. The clinical presentation often depends on the anatomic localization, rather than the size, of the tumor.
A large infiltrative tumor of the myocardium may be clinical silent, yet a small, strategically placed lesion may cause dramatic symptoms. Tumors of the left atrium that are mobile, especially myxomas, may prolapse into the mitral valve, causing obstruction of the blood flow with resulting symptoms of mitral valve disease. Cardiac angiosarcomas also have a high incidence of complications, including embolic phenomena (Butany et al 2005; Murinello et al 2007).

The neurological consequences of embolisation include transient ischemic attacks, seizures, syncope and cerebral, cerebellar, brain stem, spinal cord, or retinal infarction. The neurological event may be the first or only clinical sign of a cardiac tumor (Hart 1992; Butany et al 2005). Since new image technologies have been introduced, the source of cardioembolic stroke has been identified much more frequently. These technologies, especially TEE and, more recently, cardiovascular MRI, also allow an earlier diagnosis of cardiac tumors, notably when associated with symptoms of stroke (Brandt et al 2005; Schwab et al 2006). In the present case, differentiation between a tumor and simple thrombus was difficult with TEE because the tumor mass was small and embedded in extensive necrosis together with secondly added thrombotic material. Cardiovascular 
MRI can now provide more detailed information on the possible benign or malignant quality of the cardiac tumor (Brandt et al 2006), but noninvasive imaging techniques do not yet allow a definitive diagnosis, which can only be achieved histologically. However, the power of new imaging technologies is evident in the improvement in antemortem diagnosis in recent years. In 1968, cardiac angiosarcoma was successfully diagnosed antemortem in only four of 41 cases $(9.8 \%)$ (Glancy et al 1968). This rate increased to 55 of 67 (82.1\%) cases in 2000 (Butany and Yu 2000), and is liable to improve further.

\section{Conclusion}

This report describes an unusual cardiac angiosarcoma, localized in the left atrium, that came to the attention of clinicians due to an embolic stroke. The origin of the embolus was established as a cardiac tumor by transesophageal echocardiography, which was followed by the surgical removal of the tumor and histopathological examination. Surgical removal of the tumor was the basis for effective therapy that served to prevent recurrent embolic events, and as the initial treatment of the malignancy. Adjuvant chemotherapy completed the therapeutic regime. In summary, sensitive imaging technologies can facilitate the early detection and treatment of otherwise fatal disorders. Furthermore, the favorable outcome presented in this case underscores the benefits of interdisciplinary cooperation among physicians for effective patient care.

\section{References}

Allard MF, Taylor GP, Wilson JE, et al. 1995. Primary cardiac tumors. In: Goldhaber SZ, Braunwald E (eds). Cardiopulmonary diseases and cardiac tumors. Atlas of heart diseases. Vol. 3. Philadelphia: Current Medicine, pp. 15.1-22.
Brandt RR, Arnold R, Bohle RM, et al. 2005. Cardiac angiosarcoma: case report and review of the literature. Z Kardiol, 94:824-8.

Burke AP, Cowan D, Virmani R. 1992. Primary sarcomas of the heart. Cancer, 69: 387-95.

Burke AP, Virmani R. 1996. Atlas of tumor pathology. Tumors of the heart and great vessels. Washington: Armed Forces Institute of Pathology.

Butany J, Yu W. 2000. Cardiac angiosarcoma: two cases and a review of literature. Can J Cardiol, 16:197-205.

Butany J, Nair V, Naseemuddin A, et al. 2005. Cardiac tumours: diagnosis and management. Lancet Oncol, 6: 219-28.

Glancy DL, Morales JB, Roberts WC. 1968. Angiosarcoma of the heart. Am J Cardiol, 21:413-19.

Hart RG. 1992. Cardiogenic embolism to the brain. Lancet, 339:589-94.

Janigan DT, Husain A, Robinson NA. 1986. Cardiac angiosarcomas. A review and a case report. Cancer, 57:852-9.

Mayer F, Aebert H, Rudert M. 2007. Primary malignant sarcomas of the heart and great vessels in adult patients--a single-center experience. Oncologist, 12:1134-42.

McAllister HA Jr. 1979. Primary tumors and cysts of the heart and pericardium. Curr Probl Cardiol, 4:1-51.

Murinello A, Mendonça P, Abreu A, et al. 2007. Cardiac angiosarcoma - a review. Rev Port Cardiol, 26:577-84.

Neragi-Miandoab S, Kim J, Vlahakes GJ. 2007. Malignant tumours of the heart: a review of tumour type, diagnosis and therapy. Clin Oncol ( $R$ Coll Radiol), 19:748-56.

Putnam JB Jr, Sweeney MS, Colon R, et al. 1991. Primary cardiac sarcomas. Ann Thorac Surg, 51:906-10.

Reardon MJ, Walkes JC, Benjamin R. 2006. Therapy insight: malignant primary cardiac tumors. Nat Clin Pract Cardiovasc Med, 3:548-53.

Schwab J, Haack G, Wünsch PH, et al. 2006. Cardiac angiosarcoma: case report and review of the literature : R. R. Brandt, R. Arnold, R.M. Bohle, T. Dill, C.W. Hamm; Z Kardiol 94:824-828 (2005). Clin Res Cardiol, 95:351-2.

Yu K, Liu Y, Wang H, et al. 2007. Epidemiological and pathological characteristics of cardiac tumors: a clinical study of 242 cases. Interact Cardiovasc Thorac Surg, 6:636-9. 